\title{
IEEE 802.15.4a CSS-based Localization System for Wireless Sensor Networks
}

\author{
Jae-Eon Kim, Jihoon Kang and Daeyoung Kim \\ School of Engineering \\ Information and Communications University \\ Daejeon, Republic of Korea \\ Email: \{jekim, kunet, kimd\}@icu.ac.kr
}

\author{
Younghwoon Ko and Jungsik Kim \\ R\&D Center \\ Sensor Network Research, Inc \\ Daejeon, Republic of Korea \\ Email: \{young, kjs\}@snr.kr
}

\begin{abstract}
This demonstration shows IEEE 802.15.4a CSSbased localization system for wireless sensor networks. IEEE 802.15.4a CSS technology can provide high accurate ranging functionality to a sensor node. However, as it measures a distance based on Time-of-Flight(TOF) of RF signal, the system needs well designed ranging and report protocol. So, in this demonstration we show our ranging protocol and location calculation server.
\end{abstract}

\section{INTRODUCTION}

According to its importance as one of the most important source in several issues of realizing ubiquitous computing including context-awareness, service discovery, and so on, various localization system for Wireless Sensor Networks (WSN) have been proposed during the past several years.

IEEE 802.15.4a is an alternative PHY for IEEE 802.15.4. It can provide high accurate ranging, high aggregate throughput, and ultra low power. It has two kinds of PHY; IRUWB(Impulse Radio UWB) and CSS(Chirp Spread Spectrum). Because IR-UWB and CSS use relatively wider bandwidth than other ones, it has relative immune to multipath fading channel[7]. So, a node can measure a distance accurately.

Our localization system is based on CSS PHY for accurate ranging. At least three anchor nodes, which already knew their own positions, are needed to calculate node position. The anchor node is pre-deployed to sensing field. A node measures the distance between an anchor node and itself. After finishing distance measuring, the node sends the report packet which contains measured distance to central server. The central server calculate position of the node based on measured distance and display calculated result to a user.

Our work has several advantages against related works [2][3][4]. First of all, it uses TOA of CSS RF signal for high precision distance measurement. Most of current systems use received signal strength of RF signal which is very unstable caused by multi-path fading channel. The other advantage is centralized approach. Because we use a high performance server for calculating position, we can apply high complexity calculation algorithms and filter techniques to measured distance data for enhancing position accuracy.

The rest of this paper is organized as follows. In the next section, we introduce whole system architecture. Then Section
3 explains about the demonstration contents and finally Section 4 concludes our work and discuss about future works.

\section{System ARCHitecture}

Our localization system consists of anchor node, tag node, and central server. The anchor node knows its own position by GPS or manual setting. The tag node measures distances between near anchor nodes and itself and reports measured result to central server through sensor network. The central server calculates tag position.

Below sub-sections explain hardware and software of our localization system in detail.

\section{A. Hardware}

We can classify our hardware part to an anchor node and a tag node. Figure 1 shows the anchor and the tag node. Both anchor and tag node use $80 \mathrm{MHz}$ bandwidth (from $2.4 \mathrm{GHz}$ to $2.48 \mathrm{GHz}$ ) CSS RF signal for ranging and data communication.

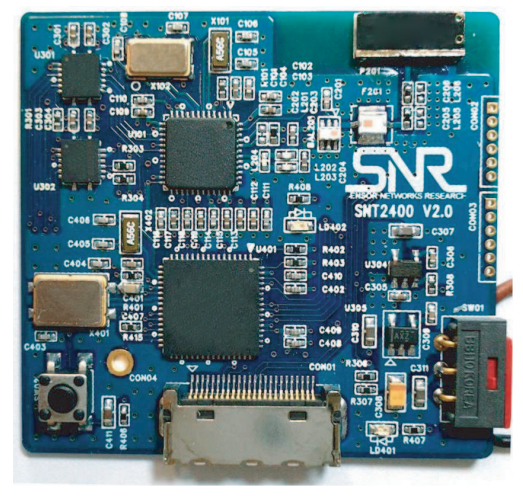

Fig. 1. Localization sensor node Hardware

The tag node has 8-bit microcontroller and RF transceiver. Additionally, it has a battery recharge circuit and a motion detect sensor. The motion detect sensor senses movement of the tag node. If the tag doesn't move, it goes to sleep mode for saving battery. The anchor and tag node are same in hardware point of view except the anchor node can choose a power source between a battery and external power source. 


\section{B. Software}

A software part of localization system consists of two parts; Tag/Anchor software and Position Calculation Server software. The Tag/Anchor software part includes ranging protocol, ranging result report protocol, and power management. The Position Calculation Server has calculation core, filters, and interfaces with database and anchor nodes.

1) Tag \& Anchor: Tag software consists of state machine, ranging engine and ranging protocol stack. State machine is main control mechanism of Tag's action in our localization system. It decides whether tag should sleep or do measurement each time. Based on state machine, IEEE 802.15.4a CSS-based ranging engine was developed. It follows CSS chirp sequence and takes accurate measurement with less $1 \mathrm{~m}$ error bound in open space.

In our system, we used two-way TOA measurement using pre-defined time interval between two TOA measurements defined in IEEE 802.15.4a. It means that during two-way measurement, nobody should send packet that occurs retransmission caused by collision. It is clearly adapted in single tag environment, but in multiple tags environment it should be totally changed. All the tags must be scheduled in centralized manner to avoid collision and scheduling policy must be fair and efficient for overall system performance.

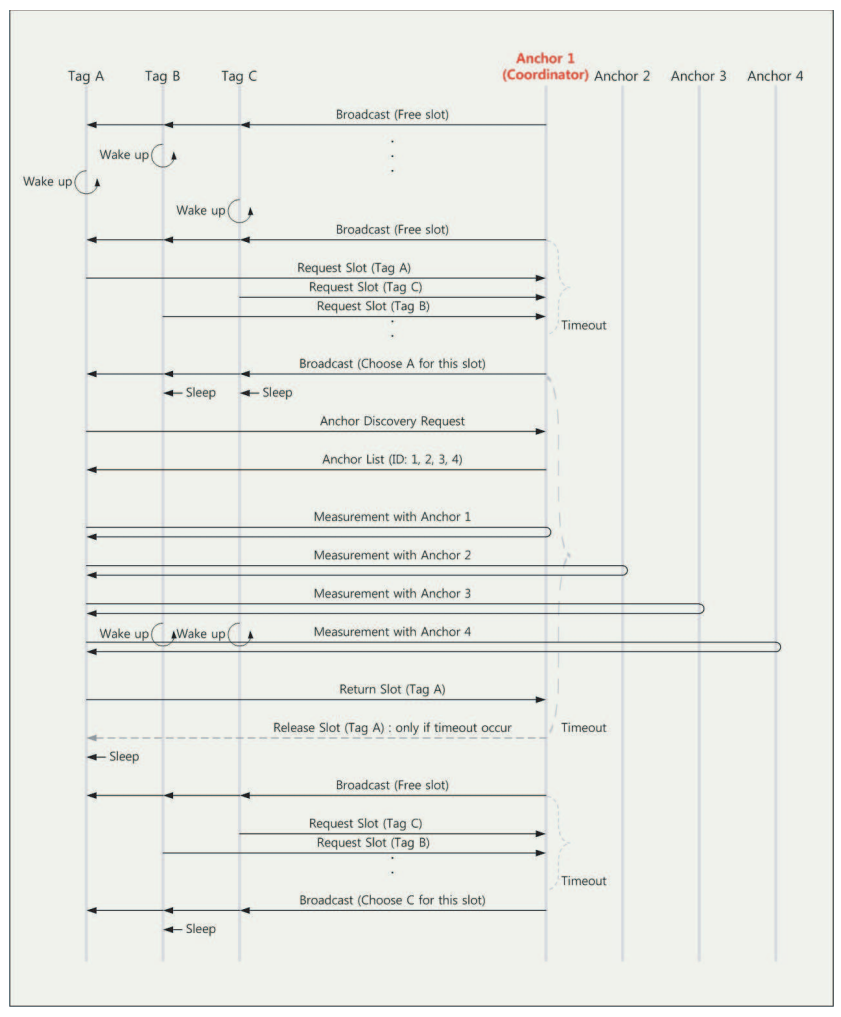

Fig. 2. Ranging Protocol

To solve this problem, we introduce ranging protocol for high density tag/anchor environment as in Figure 2. Traditionally, TDMA system was introduced to solve this kind of timing problem. However, TDMA system requires highly accurate beacon signaling and time sync with both high computing power and relatively high cost. Our ranging protocol can provide local scheduling in a cell to reduce computing complexity and costs. Among multiple anchors in a cell, we choose one anchor as network coordinator to control all timings of anchors and tags. Coordinator anchor periodically send ADV(Advertisement) packet to announce free time slot is available. Each tag receives ADV packet, it responses to get time slot by sending REQ(Request) packet. After receiving several REQ messages from tags, coordinator anchor choose one tag based on competitive voting algorithm. After coordinator anchor broadcasts ALC(Allocation) packet with voting result, un-selected tags fall into sleep during selected tag doing it's measurements with every anchors.

Just before selected tag finished its measurements, all other tags wakened up to get next time slot. Selected tag sends measurement result to coordinator anchor and gets into sleep after its measurement to avoid re-allocation for fairness. All these steps are repeated until all the tags finishes measurements.

2) Position Calculation Server: The position calculation server is central server of this localization wireless sensor network. Intuitively, it calculates the position of an tag node using the measured distance information. Figure 3 shows the central server's detail architecture.

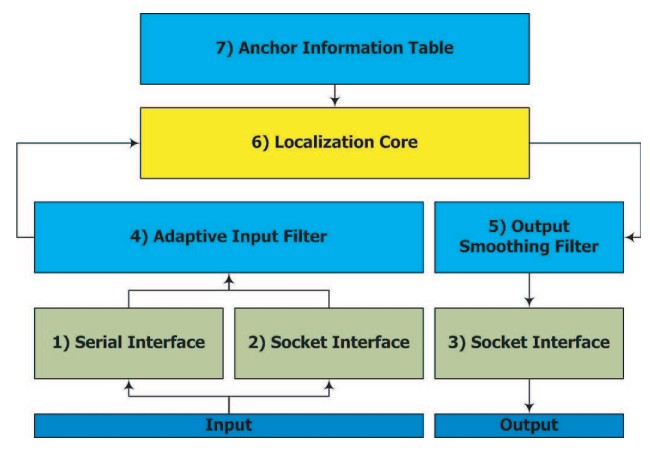

Fig. 3. Position Calculation Server Block Diagram

For calculating tag position, the central server uses multilateration as calculation core. The multi-lateration is simple and effective algorithm. It needs at least three anchor nodes for finding location and maximum nine anchor nodes can be used.

We have two kinds of filter for localization server. The first one is input filter. The filter is used for filtering irregular distance data which is from the sensor network. Our filter manages trust of each anchor nodes and gives more weight to more trustable anchor node's distance measurement results. The second filter is output smoothing filter. It smooth out sudden change which is wrong output normally. 


\section{DEMONSTRATION}

In this demonstration, we show our prototype hardware and working software. We also developed some GUI for showing current calculated position of a tag node to user.

\section{A. System Architecture}

Figure 4 represents system architecture for the conference demonstration. The demonstration includes 4 anchor nodes, 3 moving tag nodes, and central server. All anchor nodes will be installed demonstration site and position information of the anchor node will be stored to central server. The measured distance will be sent to central server through a network coordinator which is one of anchor nodes using RS232 interface.

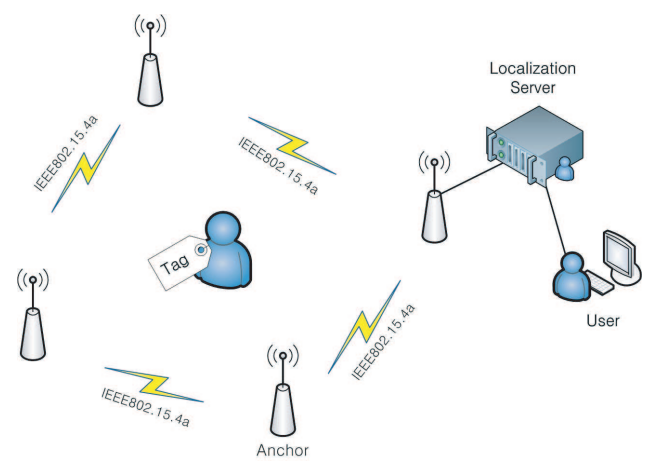

Fig. 4. Demonstration system architecture

\section{B. Demonstration Contents \& Technical Contributions}

In the demonstration, all types of anchor and tag hardware will be displayed. Prototype tag node will be equipped with battery, recharging connector and case for real deployment. Anchor node is based on ARM processor core with Ethernet, local database, and optionally CDMA interface. Anchor/Tag software architecture will be given as panel explanation. Each $\mathrm{S} / \mathrm{W}$ component will be shown in block diagram with detail explanation.

Working system consists of 4 anchors and 3 tags in a cell. Four anchors will be installed in each corners of demonstration booth and we will initially calculate its position ahead. Three multiple tags will be deployed in a demo room and it will be managed by coordinator anchor. Coordinator anchor schedule these 3 tags with $1 \mathrm{sec}$ reporting period and measurement will be refreshed in our GUI screen with accurate position. Each tag can move anywhere in a cell, it will be displayed in a screen as real-time tracking. Low-power and highly efficient localization will be shown in our demonstration.

\section{CONCLUSiOn \& Future Works}

We demonstrated IEEE 802.15.4a CSS-based localization system for wireless sensor networks. The CSS-based RF signal has relative immune to multi-path fading and provides high accurate ranging capability to a sensor node by measuring TOF

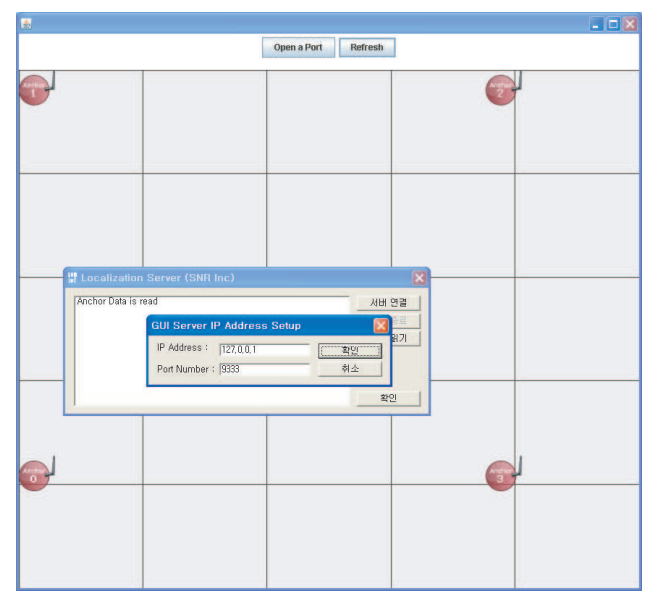

Fig. 5. Demonstration system GUI

of RF signal. However, because a tag node is asked to measure the distance with near anchor nodes one by one, the ranging protocol need to be well designed. In our work, we proposed network coordinator based a channel reservation protocol for efficient ranging protocol. The Position Calculation Server is a central server for sensor networks. It store all anchor position information for finding position of a tag node using multilateration based calculation core, adaptive trust management filter, and output smoothing filter.

As a future works, we have plan to expand out system from 4 anchor nodes to up to 100 anchor nodes and 3 tag nodes to up to $50 \mathrm{tag}$ nodes per one cell. If the number of anchor node and tag node increased, localization system will be very complex system. Therefore, especially, the ranging protocol should be very efficient.

\section{REFERENCES}

[1] N. Patwari et al, Locating the Nodes:Cooperative localization in wireless sensor networks IEEE Signal Processing Magazine, July 2005.

[2] Konrad Lorincz and Matt Welsh, MoteTrack: A Robust, Decentralized Approach to RF-Based Location Tracking Springer Personal and Ubiquitous Computing, Special Issue on Location and Context-Awareness, October 2006.

[3] DuyBach Bui, Daeyoung Kim, A Localization Algorithm with Learningbased Distances International Conference on Computer Communications, 2005.

[4] Adam Smith, Hari Balakrishnan, Michel Goraczko, Nissanka Priyantha, Tracking Moving Devices with the Cricket Location System 2nd USENIX/ACM MOBISYS Conference, Boston, MA, June 2004.

[5] Jihoon Kang, Daeyoung Kim, Sungjin Ahn, Mosaic Localization for Wireless Sensor Networks IEEE Wireless Communications and Networking Conference, Hongkong, March 2007.

[6] IEEE 802.15.4 Ranging sub-committee Final Report

[7] T. S. Rappaport, Wireless Communication Upper Saddle River, New Jersey: Prentice Hall, 1996.

[8] Daeyoung Kim, Tomas Sanchez Lopez, Seongeun Yoo, Jaeeon Kim , Sungin Ahn, Do Thu Thuy, Poh Kit Chong, Jongwoo Sung, Youngsoo Kim, Jinyoung Yang, Yoonmee Doh, ANTS platform for evolvable wireless sensor networks, Embedded Systems: Status and Perspective American Scientific publishers, 2007 